\title{
Theory and simulation of the triplet structure factor and triplet direct correlation functions in binary mixtures
}

Cite as: J. Chem. Phys. 116, 730 (2002); https://doi.org/10.1063/1.1426418

Submitted: 04 September 2001 . Accepted: 18 October 2001 . Published Online: 27 December 2001

S. Jorge, E. Lomba, and J. L. F. Abascal

ARTICLES YOU MAY BE INTERESTED IN

On the triplet structure of binary liquids

The Journal of Chemical Physics 113, 3302 (2000); https://doi.org/10.1063/1.1287337

Direct correlation function: Hard sphere fluid

The Journal of Chemical Physics 63, 601 (1975); https://doi.org/10.1063/1.431378

Triplet correlation functions for hard-spheres: Computer simulation results

The Journal of Chemical Physics 100, 5882 (1994); https://doi.org/10.1063/1.467100
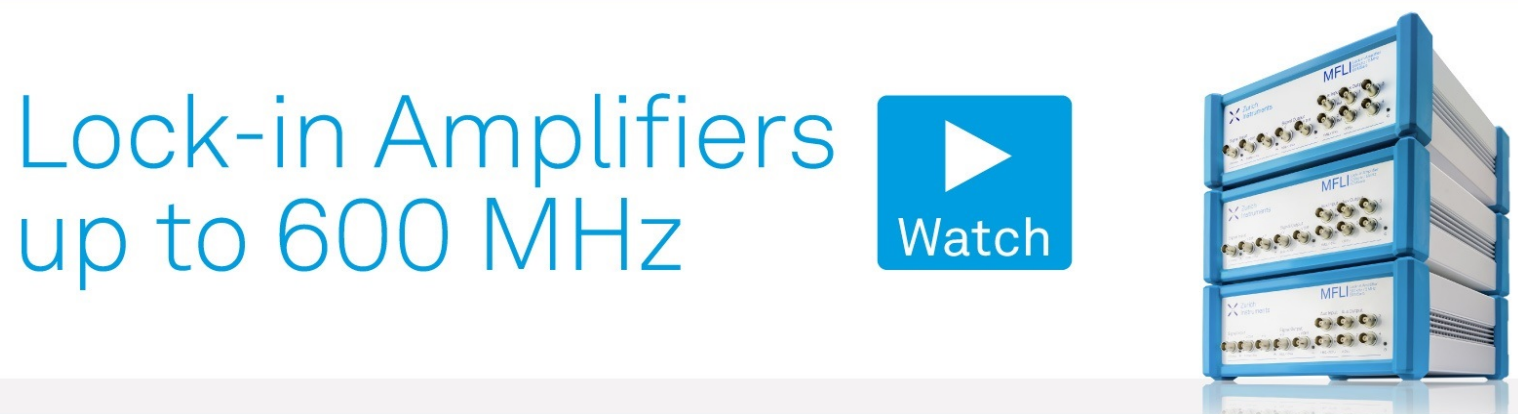

J. Chem. Phys. 116, 730 (2002); https://doi.org/10.1063/1.1426418 


\title{
Theory and simulation of the triplet structure factor and triplet direct correlation functions in binary mixtures
}

\author{
S. Jorge \\ Instituto de Química Física Rocasolano, CSIC, Serrano 119, E-28006 Madrid, Spain \\ and Departamento de Química Física I, Universidad Complutense, E-28040 Madrid, Spain \\ E. Lomba \\ Instituto de Química Física Rocasolano, CSIC, Serrano 119, E-28006 Madrid, Spain \\ J. L. F. Abascal \\ Departamento de Química Física I, Universidad Complutense, E-28040 Madrid, Spain
}

(Received 4 September 2001; accepted 18 October 2001)

\begin{abstract}
In this work we present structure factors and triplet direct correlation functions extracted from extensive Monte Carlo simulations for a binary mixture of hard spheres. The results are compared with the predictions of two integral equation theories, namely, a recently proposed extension to mixtures of Attard's inhomogeneous integral equation approach, and Barrat, Hansen, and Pastore's factorization ansatz. In general, both theories yield acceptable estimates for the triplet structure functions, though, by construction, the inhomogeneous integral equation theory is more suited to furnish triplet distribution function results, whereas the factorization ansatz provides a more handy approach to triplet direct correlation functions. (C) 2002 American Institute of Physics.
\end{abstract}

[DOI: $10.1063 / 1.1426418]$

\section{INTRODUCTION}

Triplet correlation functions play an essential role in the description of the structure and dynamics of fluids, in particular for systems whose behavior cannot be explained in terms of simple models like the pure hard sphere system or the well known Lennard-Jones fluid. Thus for instance, the triplet correlation function provided key structural information to understand the role of the Peirls distortion and chemical ordering in liquid GeTe. ${ }^{1}$ Also in a system like water, the analysis of the triplet distribution function explained how the decrease in the pair structure upon cooling near the triple point is correlated with an increase in the local tetrahedral ordering of the water molecules. ${ }^{2}$ As to dynamics, Sciortino and $\mathrm{Kob}^{3}$ have shown that the inclusion of the triple direct correlation function in the framework of the Mode Coupling Theory is essential to reproduce the wave-vector dependence of the Debye-Waller factor in supercooled silica. However, the actual calculation of the triplet correlation functions is far from being a trivial problem, neither theoretically nor in terms of computer simulation. ${ }^{4}$

The situation is even more involved when dealing with multicomponent systems. The authors, in collaboration with Kahl, ${ }^{5}$ recently presented a study of the extension to mixtures of the integral equation based on the factorization ansatz proposed by Barrat, Hansen, and Pastore (BHP). ${ }^{6}$ The comparisons performed in Ref. 5 were carried out at the level of triplet distribution functions given the scarcity of data in the literature for triplet direct correlation functions. Besides, in a recent work, ${ }^{7}$ the authors presented an extension to mixtures of Attard's inhomogeneous integral equation formalism for the calculation of three body correlation functions, ${ }^{8}$ which turned out to provide fairly good estimates for asymmetric Lennard-Jones mixtures. Still, what seems to be lack- ing in both cases is a clear assessment of the quality of both approximations as far as the mixture direct correlation function is concerned. Therefore, in this work, we present the results of extensive Monte Carlo (MC) calculations for the triplet direct correlation function and the triplet structure factor for a hard sphere mixture and perform comparisons with the theoretical approaches proposed in Refs. 5 and 7. From the triplet structure factor, which can be extracted directly from appropriate structural averages along the simulation run, it is possible to obtain the triplet direct correlation in Fourier space in a straightforward fashion.

For our inhomogeneous integral equation formalism we have chosen a self-consistent closure, the Self-Consistent Verlet's Modified approach (SCVM) ${ }^{9}$ which is known to yield excellent results at the two particle level. Following Fushiki ${ }^{10}$ the self-consistency parameters are taken from the solution of the homogeneous equation. Also here the pair distribution functions, which will be shown to be extremely accurate, are directly taken from the homogeneous equation and are not iterated to self-consistency. The inhomogeneous approach thus formulated will be denoted as inhomogeneous SCVM (ISCVM). We will see that both the BHP theory using as input the density derivatives of the pair correlation function provided by the SCVM theory and the ISCVM approach provide a rather accurate picture of the three body direct correlation functions and triplet structure factors. Moreover, the ISCVM triplet distribution function is particularly accurate. For this property, the BHP results are plagued with the some spurious effects stemming from the inverse Fourier transformation of a discontinuous function. But, in general, aside from these numerical uncertainties in the Fourier transforms, the accuracy of both approaches is comparable. 
The rest of the paper is organized as follows: Sec. II is devoted to a brief presentation of the simulation details. Section III contains a summary of the inhomogeneous integral equation approach. The essentials of the BHP ansatz in the mixture case are collected in Sec. IV. And finally, our most significant results are presented and commented upon in Sec. V.

\section{SIMULATION OF TRIPLET CORRELATION FUNCTIONS}

The MC simulation technique has been used to study a binary equimolar hard-sphere mixture with packing fraction $\eta=(\pi / 6)(\rho / 2)\left(\sigma_{\alpha \alpha}^{3}+\sigma_{\beta \beta}^{3}\right)=0.4-\rho$ being the total number density-and a size ratio $\sigma_{\alpha \alpha} / \sigma_{\beta \beta}=0.8$. The structure factors $S_{\mu \nu}^{(2)}(\mathbf{k})$ and $S_{\mu \nu \xi}^{(3)}\left(\mathbf{k}, \mathbf{k}^{\prime}\right)$ are calculated evaluating the corresponding averages, ${ }^{11}$

$$
\begin{aligned}
& S_{\mu \nu}^{(2)}(\mathbf{k})=\frac{1}{N}\left\langle\rho_{\mathbf{k}}^{\mu} \rho_{-\mathbf{k}}^{\nu}\right\rangle, \\
& S_{\mu \nu \xi}^{(3)}\left(\mathbf{k}, \mathbf{k}^{\prime}\right)=\frac{1}{N}\left\langle\rho_{\mathbf{k}}^{\mu} \rho_{\mathbf{k}^{\prime}}^{\nu} \rho_{-\mathbf{k}-\mathbf{k}^{\prime}}^{\xi}\right\rangle,
\end{aligned}
$$

where $N$ is the particle number, and the Fourier components of the density $\rho(\mathbf{r})=\Sigma_{i} \delta\left(\mathbf{r}-\mathbf{r}_{i}\right)$ are

$$
\rho_{\mathbf{k}}=\sum_{i}^{N} e^{-i \mathbf{k r}_{i}}
$$

Using the pair and triplet structure factors, the triplet direct correlation functions $\widetilde{c}_{\mu \nu \xi}^{(3)}\left(\mathbf{k}, \mathbf{k}^{\prime}\right)$ can be calculated by solving a linear system of equations, derived from the triplet Ornstein-Zernike equation,

$$
\begin{aligned}
S_{\mu \nu \xi}^{(3)}\left(\mathbf{k}, \mathbf{k}^{\prime}\right)= & \sum_{\epsilon \sigma \eta} S_{\mu \epsilon}^{(2)}(k) S_{\nu \sigma}^{(2)}\left(k^{\prime}\right) S_{\xi \eta}^{(2)}\left(\left|\mathbf{k}+\mathbf{k}^{\prime}\right|\right) \\
& \times\left(\frac{1}{x_{\epsilon}^{2}} \delta_{\epsilon \sigma} \delta_{\epsilon \eta}+\rho^{2} \widetilde{c}_{\epsilon \sigma \eta}^{(3)}\left(\mathbf{k}, \mathbf{k}^{\prime}\right)\right) .
\end{aligned}
$$

It should be pointed out here that in Ref. 5 in the corresponding equation [Eq. (15)] the indices of the pair functions are misplaced and as a consequence the results presented therein are affected by minor errors, as will be shown in the following sections. For the sake of simplicity only isosceles triangle configurations corresponding to $|\mathbf{k}|=\left|\mathbf{k}^{\prime}\right|=k$ and $\cos \theta=\mathbf{k k}^{\prime} /\left|\mathbf{k} \mathbf{k}^{\prime}\right|$ have been analyzed, and therefore the maximum value of $\left|\mathbf{k}+\mathbf{k}^{\prime}\right|$ is $2 k$ (see Fig. 1). For a 500 particles sample, the simulation has been run along 6.3 $\times 10^{7}$ steps, which is of the order of the simulations recently carried out to evaluate triplet correlations in liquid silica. ${ }^{3}$ Statistical averages have been taken over $2.6 \times 10^{6}$ configurations, and standard errors have also been estimated using block averages, leading to variances below $0.4 \%$ for the pair structure factor, around $10 \%$ for the triplet structure factor, and ranging from 1 to $30 \%$ for $\widetilde{c}_{\mu \nu \xi}^{(3)}\left(\mathbf{k}, \mathbf{k}^{\prime}\right)$.

\section{THE INHOMOGENEOUS INTEGRAL EQUATION}

The inhomogeneous integral equation approach proposed by Attard ${ }^{8}$ was already discussed for mixtures in Ref. 7, where it was applied to study a binary Lennard-Jones fluid

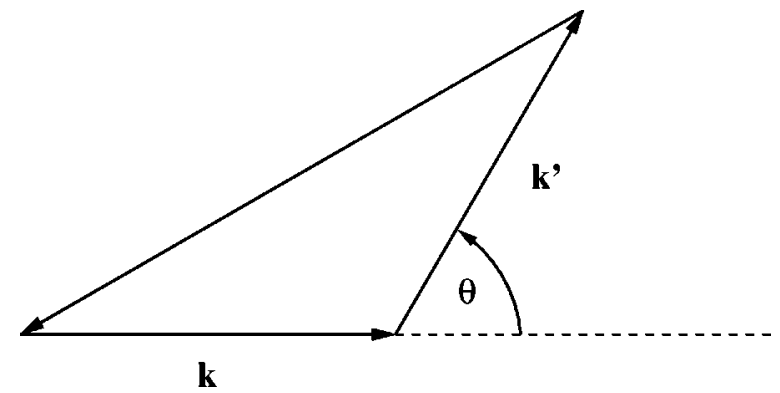

FIG. 1. Isosceles triangle configuration in $k$-space.

mixture. The theory analyzes the triplet structure of the fluid considering one of the particles of a given triplet as a source of an external field. Therefore, the Ornstein-Zernike (OZ) integral equation becomes inhomogeneous (IOZ), and for a mixture of $n$ components reads

$$
\begin{aligned}
h_{\mu \nu \underline{\gamma}}^{(2)}\left(r_{1}, r_{2}, \theta_{12}\right)= & c_{\mu \nu \underline{\gamma}}^{(2)}\left(r_{1}, r_{2}, \theta_{12}\right) \\
& +\sum_{\lambda=1}^{n} \int d r_{4} \rho_{\lambda \underline{\gamma}}\left(r_{4}\right) c_{\mu \lambda \underline{\gamma}}^{(2)}\left(r_{1}, r_{4}, \theta_{14}\right) \\
& \times h_{\lambda \nu \underline{\underline{\gamma}}}^{(2)}\left(r_{4}, r_{2}, \theta_{42}\right),
\end{aligned}
$$

being $h_{\mu \nu \gamma}^{(2)}\left(\mathbf{r}_{1}, \mathbf{r}_{2}\right)$ the pair total correlation function and $c_{\mu \nu \gamma}^{(2)}\left(\mathbf{r}_{1}, \mathbf{r}_{2}\right)$ the pair direct correlation function. Coordinates $\mathbf{r}_{1}$ and $\mathbf{r}_{2}$ correspond to particles $\mu$ and $\nu$, respectively, and $\cos \theta_{i j}=\mathbf{r}_{i} \mathbf{r}_{j} / r_{i} r_{j}$.

This approach considers the source particle $(\chi)$ as an inhomogeneous perturbation in the system, and consequently instead of the homogeneous density $\rho_{\lambda}$ we will have a density profile $\rho_{\lambda \underline{\gamma}}(r)$ around $\gamma$. This density profile is actually connected to the homogeneous pair distribution function through $\rho_{\lambda \gamma}(r)=\rho_{\lambda} g_{\lambda \gamma}(r)$. As done in Ref. 7, it is possible to obtain the density profile from the three particle distribution functions by means of the Triezenberg-ZwanzigWertheim-Lovett-Mou-Buff (TZWLMB) (Refs. 12，13)

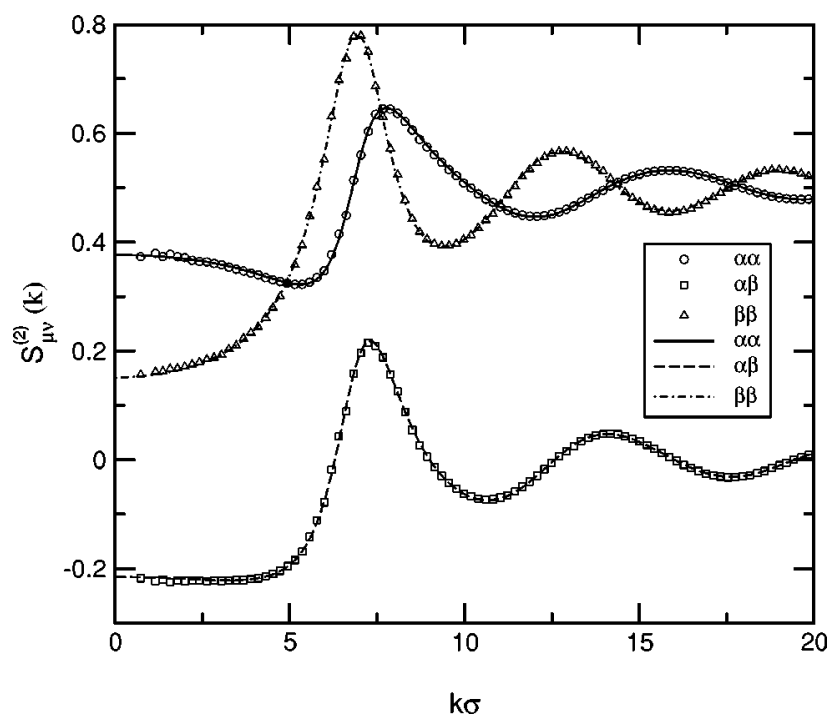

FIG. 2. Pair structure factors $S_{\mu \nu}^{(2)}(k)$ for a hard sphere mixture calculated from simulation (symbols) and the SCVM integral equation (lines). 


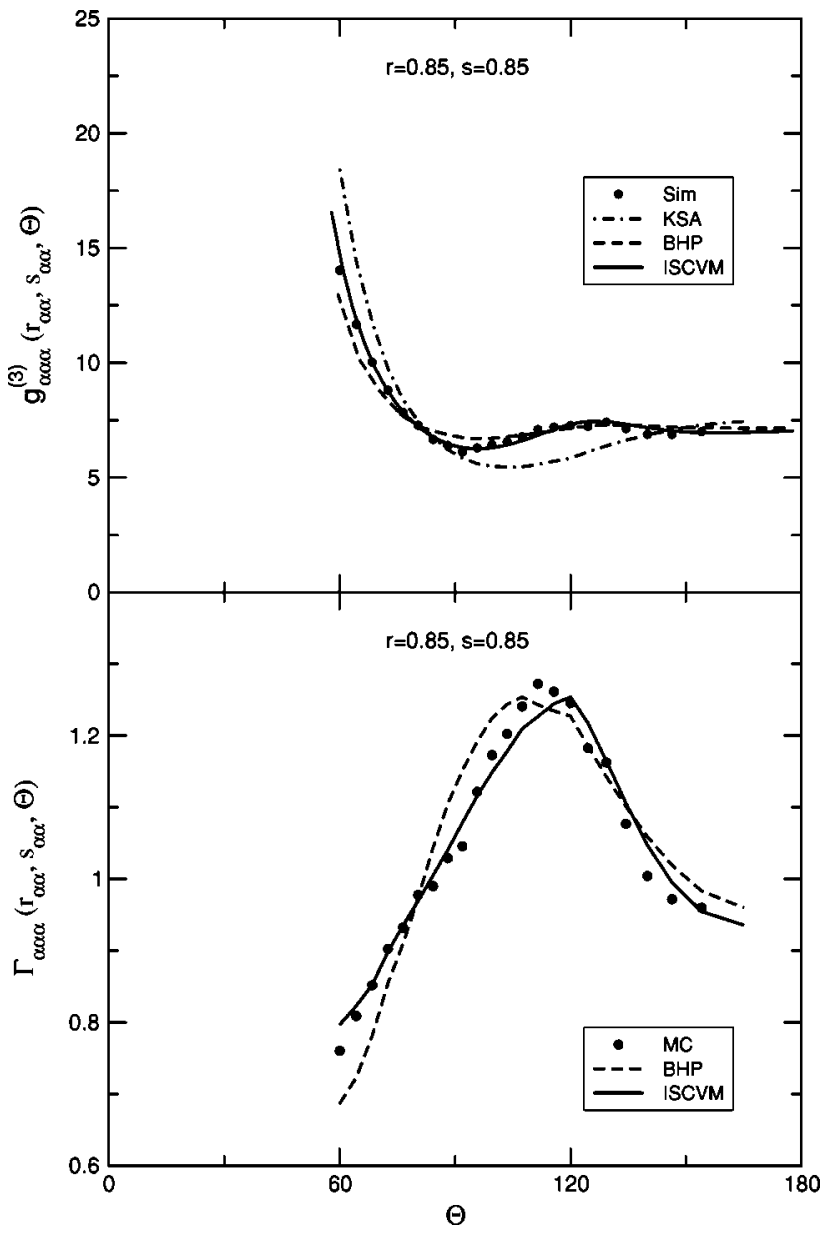

FIG. 3. Triplet distribution function $g_{\alpha \alpha \alpha}^{(3)}\left(r_{\alpha \alpha}, s_{\alpha \alpha}, \theta\right)$ and $\Gamma_{\alpha \alpha \alpha}\left(r_{\alpha \alpha}, s_{\alpha \alpha}, \theta\right)$ between like particles in an isosceles configuration for a hard sphere mixture. The source particle in the ISCVM approach is the smallest particle $\alpha$.

equation and recalculate those triplet functions with a new density profile, iterating until convergence. In this work, the high quality of the density profiles obtained from the SCVM equation, ${ }^{9}$ enables their use as a direct input for Eq. (5). No iteration will be used since these profiles may be considered essentially exact.

The IOZ equation is complemented with a closure relation which relates the interaction potential $v_{\mu \nu}\left(r_{12}\right)$ to the pair correlation functions. It can be formally written as follows:

$$
\begin{aligned}
g_{\mu \nu \underline{\gamma}}^{(2)}\left(r_{1}, r_{2}, \theta_{12}\right)= & \exp \left\{-\beta v_{\mu \nu}\left(r_{12}\right)+h_{\mu \nu \underline{\gamma}}^{(2)}\left(r_{1}, r_{2}, \theta_{12}\right)\right. \\
& -c_{\mu \nu \underline{\gamma}}^{(2)}\left(r_{1}, r_{2}, \theta_{12}\right) \\
& \left.+B_{\mu \nu \underline{\gamma}}\left(r_{1}, r_{2}, \theta_{12}\right)\right\}
\end{aligned}
$$

where $\beta=1 / K_{B} T, r_{12}=\left(r_{1}^{2}+r_{2}^{2}-2 r_{1} r_{2} \cos \theta_{12}\right)^{1 / 2}$, and $B_{\mu \nu \gamma}$ is the bridge function. In our calculations we have used the bridge function corresponding to the SCVM (Ref. 9) closure which implements certain degree of thermodynamic consistency. Namely,

$$
B_{\mu \nu \underline{\gamma}}\left(r_{1}, r_{2}, \theta_{12}\right)=-\frac{\Phi_{\mu \nu}(r, \psi) s_{\mu \nu \underline{\gamma}}(r)^{2}}{2\left[1+\Omega_{\mu \nu} s_{\mu \nu \underline{\gamma}}(r)\right]},
$$

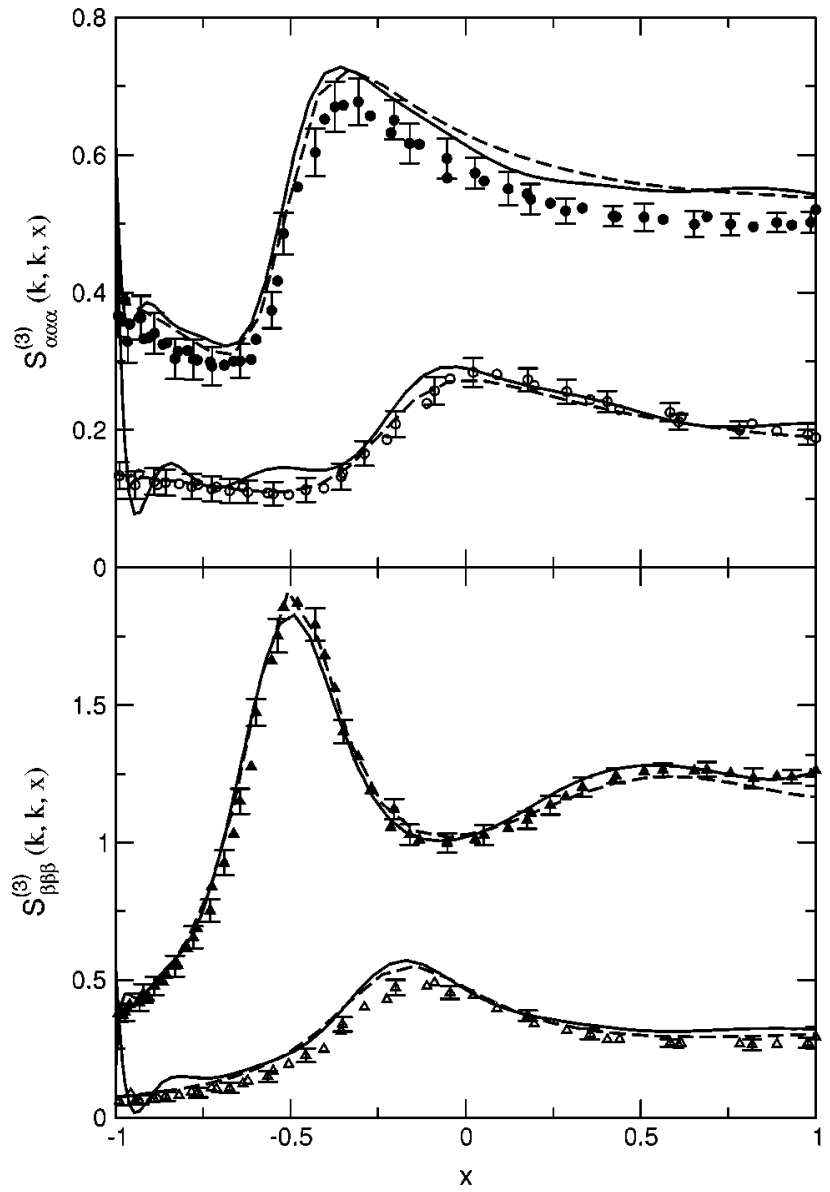

FIG. 4. Triplet structure factor for an isosceles configuration of like particles $S_{\alpha \alpha \alpha}^{(3)}(k, k, x)$ and $S_{\beta \beta \beta}^{(3)}(k, k, x)$ vs $x=\cos \theta$. Open symbols denote simulation results for $k \sigma=5.46$ and solid symbols correspond to $k \sigma=6.93$. Dashed curves represent BHP results and solid curves the ISCVM.

where $\Omega_{\mu \nu}=\omega\left(1.1-\rho \sigma_{\mu \nu}^{3} / 3\right)$ and $s_{\mu \nu \gamma}=h_{\mu \nu \gamma}-c_{\mu \nu \gamma}$ is the indirect correlation function. The interpolation function can be expressed as

$$
\Phi_{\mu \nu}\left(r_{1}, r_{2}, \theta_{12} ; \psi\right)=1+\left[1+\tanh \left(r_{12}-\sigma_{\mu \nu}\right)\right] \frac{\psi-1}{2},
$$

where the parameters $\psi$ and $\omega$ are taken from the solution of the homogeneous equation ${ }^{7,10,14}$ in which consistency between virial and fluctuation theorem compressibility, and also between chemical potential and virial pressure is imposed.

From a practical point of view, in order to solve the IOZ equation the three particle functions are expanded in Legendre polynomials to obtain

$$
\begin{aligned}
\hat{h}_{m}^{\mu \nu \nu \underline{\gamma}}\left(r_{1}, r_{2}\right)= & \hat{c}_{m}^{\mu \nu \underline{\gamma}}\left(r_{1}, r_{2}\right) \\
& +\frac{4 \pi}{2 m+1} \sum_{\lambda=1}^{n} \int d r_{4} r_{4}^{2} \rho_{\lambda \underline{\gamma}}\left(r_{4}\right) \\
& \times \hat{c}_{m}^{\mu \lambda \underline{\gamma}}\left(r_{1}, r_{4}\right) \hat{h}_{m}^{\lambda \nu \gamma}\left(r_{4}, r_{2}\right),
\end{aligned}
$$

with the hatted quantities representing the coefficients of the inhomogeneous functions in the Legendre series and $m$ 


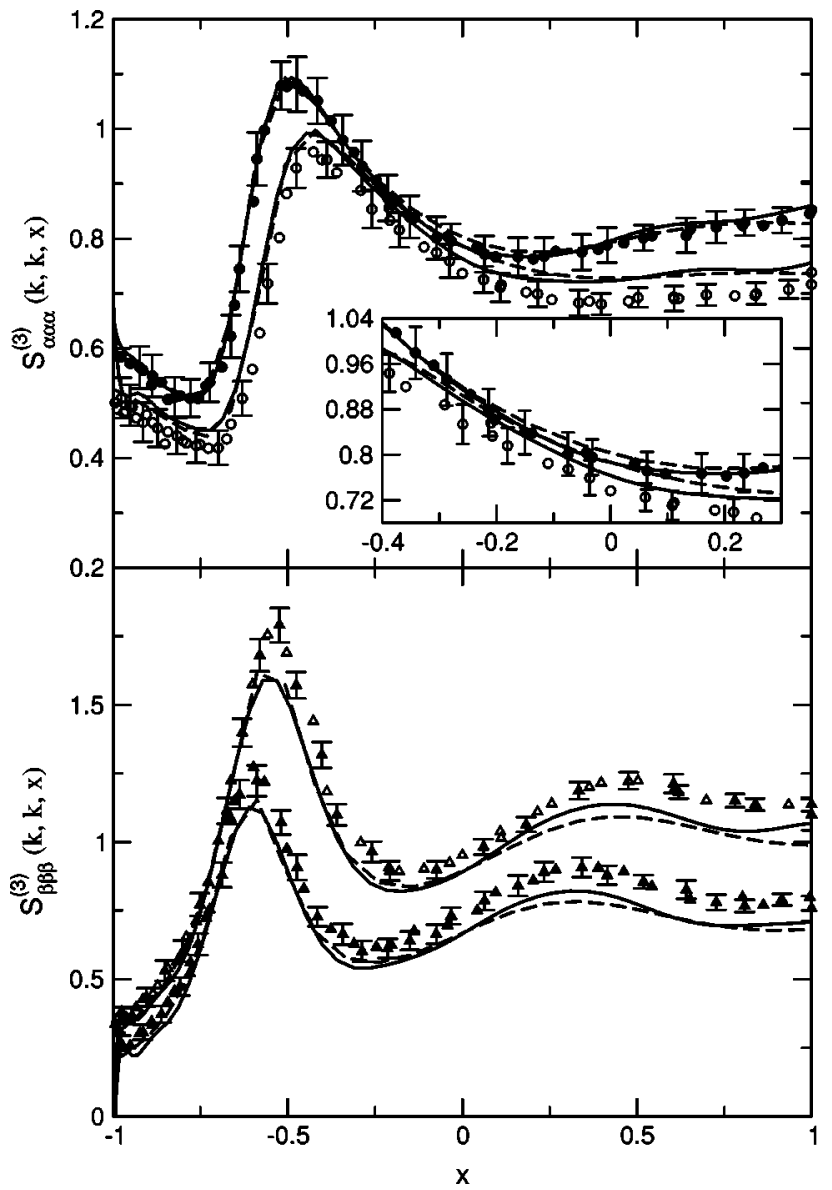

FIG. 5. Same as Fig. 4 for $k \sigma=7.35$ (empty symbols) and $k \sigma=7.77$ (solid symbols).

denotes the order of the Legendre polynomial. The hard core discontinuities introduce additional difficulties to evaluate the Legendre transforms. To cope with this we have followed the procedure suggested by Attard $^{8}$ which is summarized in the Appendix for completeness, since some misprints have been found in the expressions of Ref. 8. In order to solve Eqs. (5) and (6), equations with different source particles are decoupled as in Ref. 7. Also now the Generalized Minimal RESidual algorithm for nonlinear systems of equations (GMRESNL) (Refs. 7, 15, 16) has been used to solve each coupled system of equations. The homogeneous SCVM pair functions have been discretized on a grid of 2048 points with mesh size $0.0125 \sigma$ ( $\sigma$ being the biggest particle diameter). The number of grid points on the radial coordinate was set to 250, and the discrete Legendre transforms have been carried out with 64 angular nodes.

From the solution of Eqs. (5) and (6) one gets the inhomogeneous pair distribution function, which relates to the triplet distribution function through

$$
\begin{aligned}
g_{\mu \nu \underline{\gamma}}^{(3)}\left(r_{1}, r_{2}, \cos \theta_{12}\right)= & g_{\mu \gamma}\left(r_{1}\right) g_{\nu \gamma}\left(r_{2}\right) \\
& \times g_{\mu \nu \underline{\gamma}}^{(2)}\left(r_{1}, r_{2}, \cos \theta_{12}\right) .
\end{aligned}
$$

In turn, the triplet total correlation function is given by

$$
\begin{aligned}
h_{\mu \nu \gamma}^{(3)}\left(\mathbf{r}, \mathbf{r}^{\prime}\right)= & g_{\mu \nu \gamma}^{(3)}\left(\mathbf{r}, \mathbf{r}^{\prime}\right)-h_{\mu \gamma}(r)-h_{\nu \gamma}\left(r^{\prime}\right) \\
& -h_{\mu \nu}\left(\left|\mathbf{r}+\mathbf{r}^{\prime}\right|\right)-1,
\end{aligned}
$$

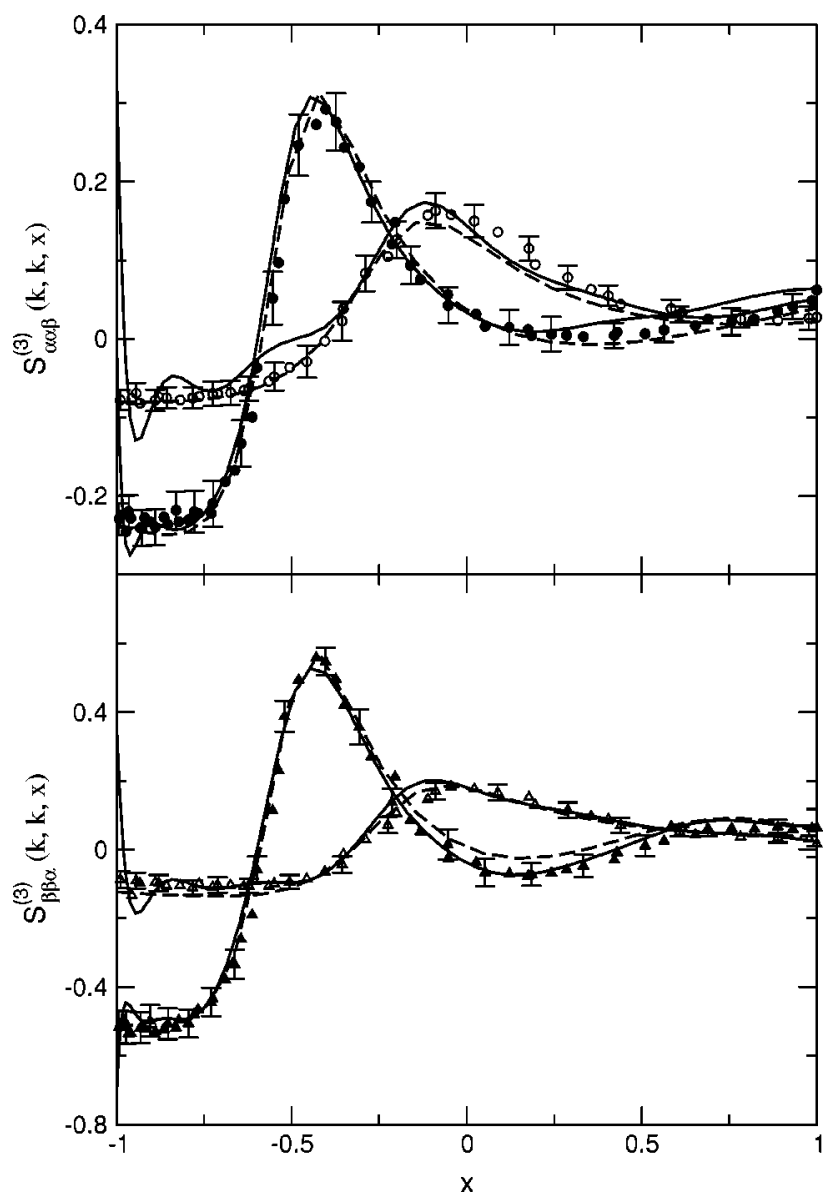

FIG. 6. Triplet structure factor for an isosceles configuration of unlike particles $S_{\alpha \alpha \beta}^{(3)}(k, k, x)$ and $S_{\beta \beta \alpha}^{(3)}(k, k, x)$ vs $x=\cos \theta$. Legend as in Fig. 4.

which relates with the sought triplet structure factor via

$$
\begin{aligned}
S_{\mu \nu \gamma}^{(3)}\left(\mathbf{k}, \mathbf{k}^{\prime}\right)= & \delta_{\mu \nu} \delta_{\mu \gamma} x_{\mu}+\delta_{\mu \gamma} x_{\mu} x_{\nu} \rho \widetilde{h}_{\mu \nu}\left(k^{\prime}\right) \\
& +\delta_{\nu \gamma} x_{\mu} x_{\gamma} \rho \widetilde{h}_{\mu \gamma}(k) \\
& +\delta_{\mu \nu} x_{\nu} x_{\gamma} \rho \widetilde{h}_{\nu \gamma}\left(\left|\mathbf{k}+\mathbf{k}^{\prime}\right|\right)+x_{\mu} x_{\nu} x_{\gamma} \rho^{2} \\
& \times \int e^{-i \mathbf{k} \mathbf{r}_{\mu}} e^{-i \mathbf{k}^{\prime} \mathbf{r}_{\nu}^{\prime} h_{\mu \nu \gamma}^{(3)}\left(\mathbf{r}, \mathbf{r}^{\prime}\right) d \mathbf{r} d \mathbf{r}^{\prime}}
\end{aligned}
$$

From this, once more, the triplet direct correlation function can be evaluated by means of Eq. (4).

\section{THE BHP THEORY}

The theory of Barrat, Hansen, and Pastore ${ }^{6}$ assumes a factorization ansatz for the triplet direct correlation function $c_{\mu \nu \xi}^{(3)}\left(\mathbf{r}, \mathbf{r}^{\prime}\right)$ of the form,

$$
c_{\mu \nu \xi}^{(3)}\left(\mathbf{r}, \mathbf{r}^{\prime}\right)=t_{\mu \nu}^{\mu \nu \xi}\left(\left|\mathbf{r}-\mathbf{r}^{\prime}\right|\right) t_{\mu \xi}^{\mu \nu \xi}(r) t_{\nu \xi}^{\mu \nu \xi}\left(r^{\prime}\right) .
$$

The $t_{\mu \nu}^{\mu \nu \xi}(r)$ function of the ansatz can be calculated from the sum-rule,

$$
\begin{aligned}
\frac{\partial c_{\mu \xi}^{(2)}(r)}{\partial \rho_{\nu}} & =\int c_{\mu \nu \xi}^{(3)}\left(\mathbf{r}, \mathbf{r}^{\prime}\right) d \mathbf{r}^{\prime} \\
& =t_{\mu \xi}^{\mu \nu \xi}(r) \int t_{\nu \xi}^{\mu \nu \xi}\left(r^{\prime}\right) t_{\mu \nu}^{\mu \nu \xi}\left(\left|\mathbf{r}-\mathbf{r}^{\prime}\right|\right) d \mathbf{r}^{\prime},
\end{aligned}
$$




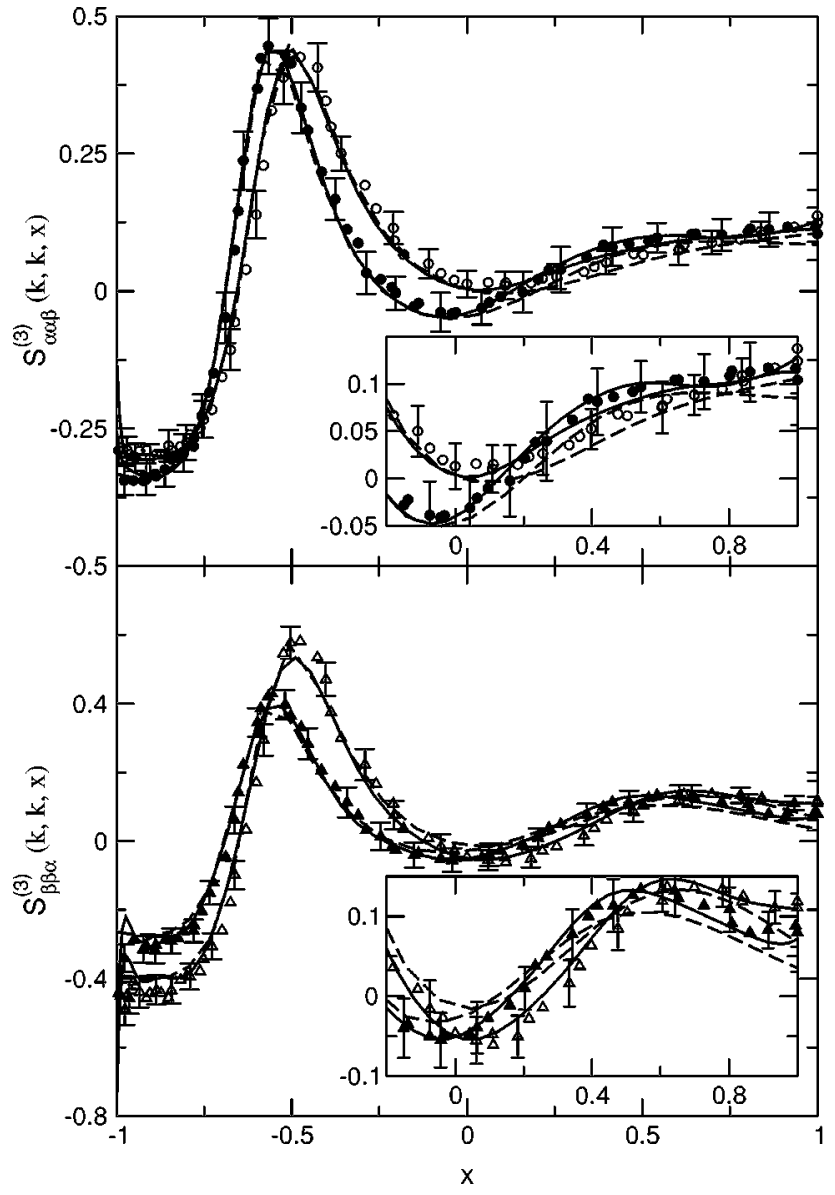

FIG. 7. Same as Fig. 6 for $k \sigma=7.35$ (empty symbols) and $k \sigma=7.77$ (solid symbols).

which in $k$-space reads

$$
\frac{\partial \widetilde{c}_{\mu \xi}^{(2)}(k)}{\partial \rho_{\nu}}=\widetilde{c}_{\mu \nu \xi}^{(3)}\left(\mathbf{k}, \mathbf{k}^{\prime}=0\right) .
$$

The derivatives of the direct correlation function are numerically evaluated from the results of the SCVM equation for the mixture and are the only input required. Once the $t_{\mu \nu}^{\mu \nu \xi}(r)$ functions have been calculated, they are Fouriertransformed and used to calculate $\widetilde{c}_{\mu \nu \xi}^{(3)}\left(\mathbf{k}, \mathbf{k}^{\prime}\right)$. Introducing $\widetilde{c}_{\mu \nu \xi}^{(3)}\left(\mathbf{k}, \mathbf{k}^{\prime}\right)$ in the triplet OZ integral equation [see Eq. (17) in Ref. 5] one can obtain $\widetilde{h}_{\mu \nu \xi}^{(3)}\left(\mathbf{k}, \mathbf{k}^{\prime}\right)$ and, after an inverse Fourier transform, the three particle total correlation function, $h_{\mu \nu \xi}^{(3)}\left(\mathbf{r}, \mathbf{r}^{\prime}\right) . S_{\mu \nu \xi}^{(3)}\left(\mathbf{k}, \mathbf{k}^{\prime}\right)$ is easily obtained from the Fourier transformed triplet direct correlation function using Eq. (4).

All the numerical details relative to the calculations within the BHP formalism can be found in Ref. 5 .

\section{RESULTS}

As mentioned before, one of the key input quantities in the theoretical approaches dealt with herein, is the pair structure of the homogeneous fluid. In Fig. 2 we have plotted the partial structure factors obtained from the simulation averages and the SCVM results. It is immediately evident that the agreement is practically perfect. Consequently, we are confi-

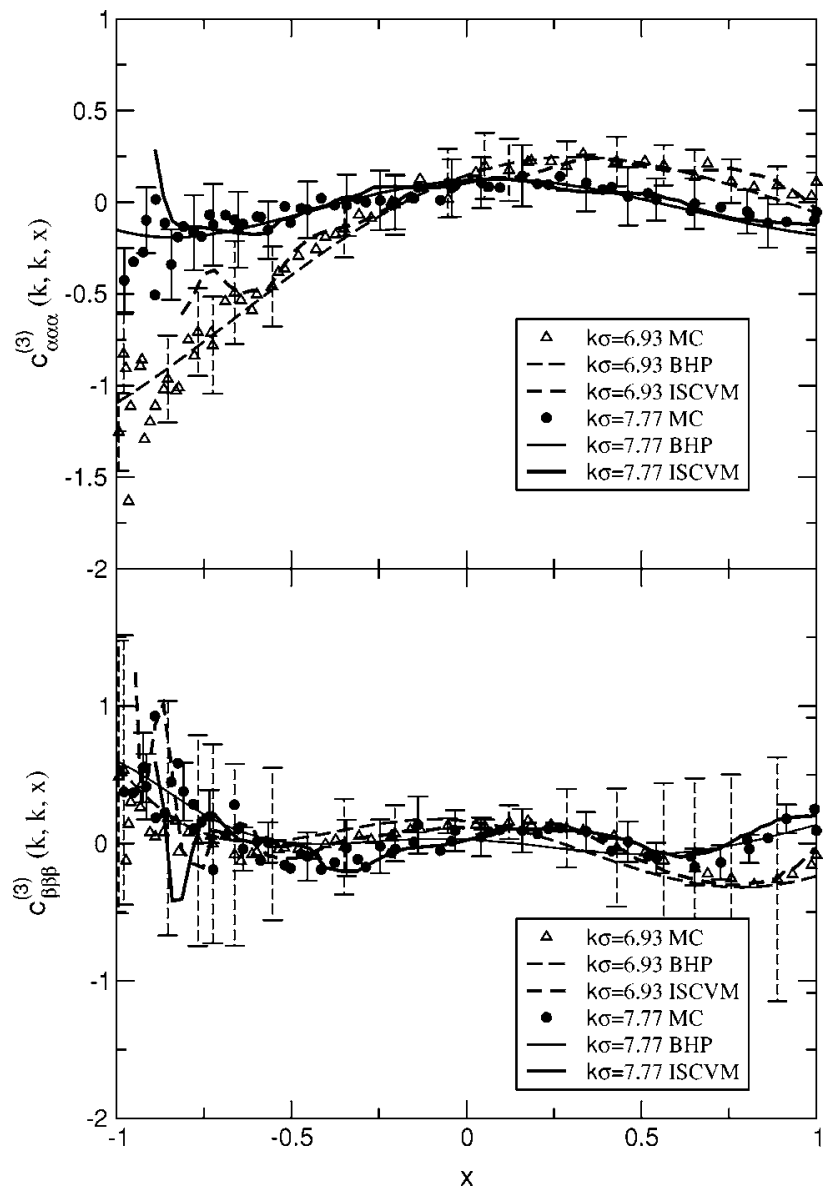

FIG. 8. Angular dependence of Fourier transform of the triplet direct correlation function for an isosceles configuration of like particles $c_{\alpha \alpha \alpha}^{(3)}(k, k, x)$ and $c_{\beta \beta \beta}^{(3)}(k, k, x)$.

dent to use both the SCVM pair correlation functions as input for the ISCVM equation and to provide the derivatives of the SCVM direct correlation function within the BHP formalism.

Among the triplet distribution functions obtained from theory and simulation, only that corresponding to like particles is illustrated in Fig. 3 for isosceles configurations. The BHP data presented here are in better agreement with MC than those of Ref. 5 due to the correction of Eq. (4). As pointed out by Bildstein and Kahl ${ }^{17}$ for the single component case, the most accurate results are produced by the inhomogeneous integral equation approach, i.e., by the ISCVM in our case. Note however, that the BHP results are plagued with the inaccuracies of the inverse Fourier transformation which is a highly nontrivial numerical problem in the case of inhomogeneous functions. Also, as found in Ref. 7 the results of the ISCVM approach are considerably less accurate when the largest particle is taken as source particle. Once again, we might well think that the largest size implies a larger perturbation potential in the inhomogeneous integral equation-let us recall here that the integral equation is decoupled according to the type of source particle used. It has also to be mentioned, that despite the inaccuracies stemming from the inverse Fourier transform, the ISCVM $g^{(3)} s$ are in somewhat better agreement with $\mathrm{MC}$ than the corresponding results obtained in Ref. 7 for Lennard-Jones systems using a 


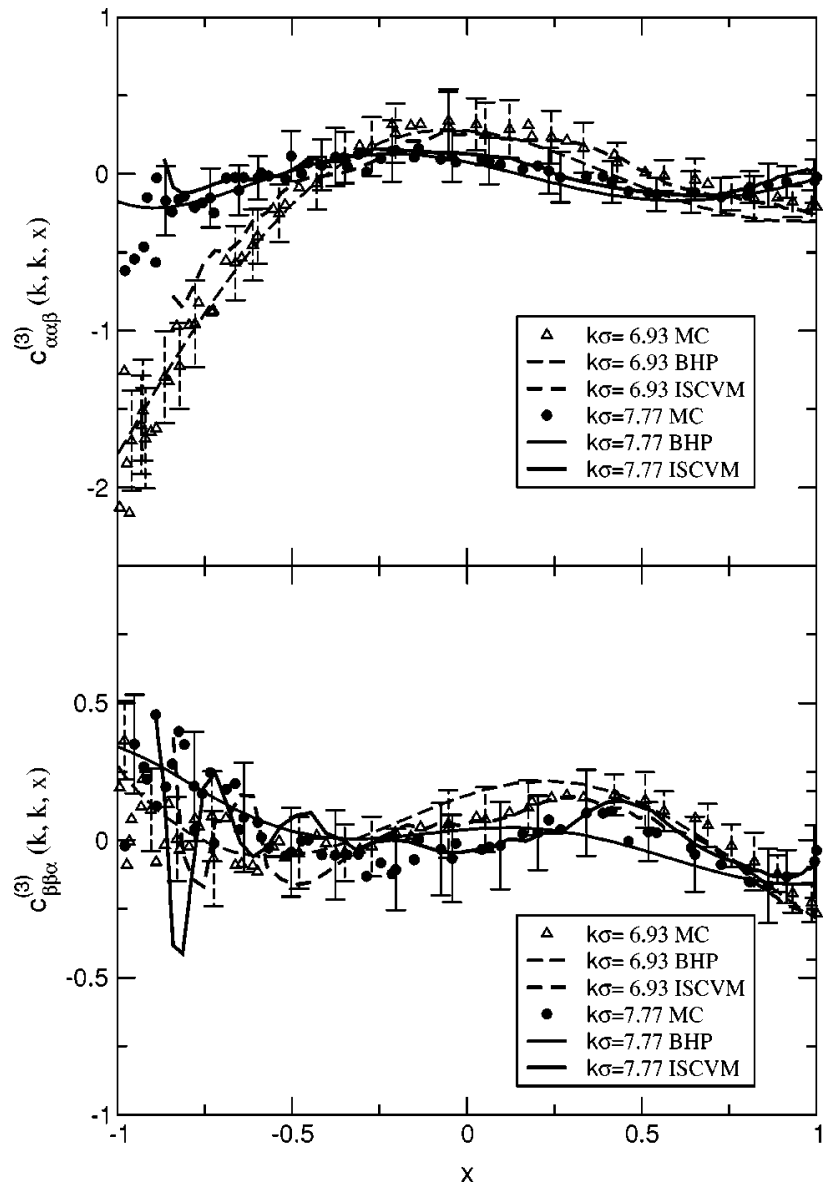

FIG. 9. Angular dependence of the Fourier transform of the triplet direct correlation function for an isosceles configuration of unlike particles $c_{\alpha \alpha \beta}^{(3)}(k, k, x)$ and $c_{\beta \beta \alpha}^{(3)}(k, k, x)$.

Hybrid Mean Spherical (HMSA) closure. This can easily be understood since the double consistency criterion imposed on the SCVM makes this approach considerably more accurate for hard sphere systems. ${ }^{9}$
As to the triplet structure factor, this is depicted in Figs. $4-7$. One sees that both theoretical approaches yield results almost within the statistical uncertainties of the simulation. However, one observes that for given configurations the ISCVM results exhibit certain artificial wiggles, which stem from the Fourier transform procedure. The only alternative to suppress these wiggles is to increase the number of radial coordinates and decrease the angular grid in the inhomogeneous functions, however, the calculations presented herein are at the limit of the available computational facilities. Nonetheless, as a whole, one would be tempted to say that both approaches have comparable accuracy.

Finally, in Figs. 8 and 9 we have depicted the results of $\widetilde{c}_{\mu \nu \xi}^{(3)}(k, k, x)$ for different configurations. We can see that the theoretical approaches yield results practically within the statistical uncertainties. Again here, the ISCVM results present the characteristic wiggles resulting from the Fourier transform, and these are magnified in the case of $\widetilde{c}_{\mu \nu \xi}^{(3)}\left(\mathbf{k}, \mathbf{k}^{\prime}\right)$ due to the way in which this quantity is obtained. This can be easily understood by examining the expressions that relate $\widetilde{c}^{(3)}\left(\mathbf{k}, \mathbf{k}^{\prime}\right)$ and $S^{(3)}\left(\mathbf{k}, \mathbf{k}^{\prime}\right)$ in the one component case.

In Fig. 10 is illustrated the $k$ dependence of the Fourier transform of the triplet direct correlation for isosceles configurations of like and unlike particles at given configurations. One readily sees that the BHP theory performs rather well for all conditions. Note that the simulation value for the configuration with $\cos \theta=-1$ has been directly evaluated from the sum rule (15). The corresponding ISCVM functions have not been included because for small $k$ values the spurious wiggles distort considerably the $\widetilde{c}_{\mu \nu \xi}^{(3)}\left(\mathbf{k}, \mathbf{k}^{\prime}\right)$ results, although quite good agreement is found for larger $k$ values.

In summary, we have seen that both the inhomogeneous integral approach in its ISCVM formulation and the BHP ansatz provide a reliable alternative for the calculation of triplet correlations, provided the pair structure is known. The ISCVM approach, however, can be coupled to a TZWLMB equation and thus the requirement of an a priori knowledge

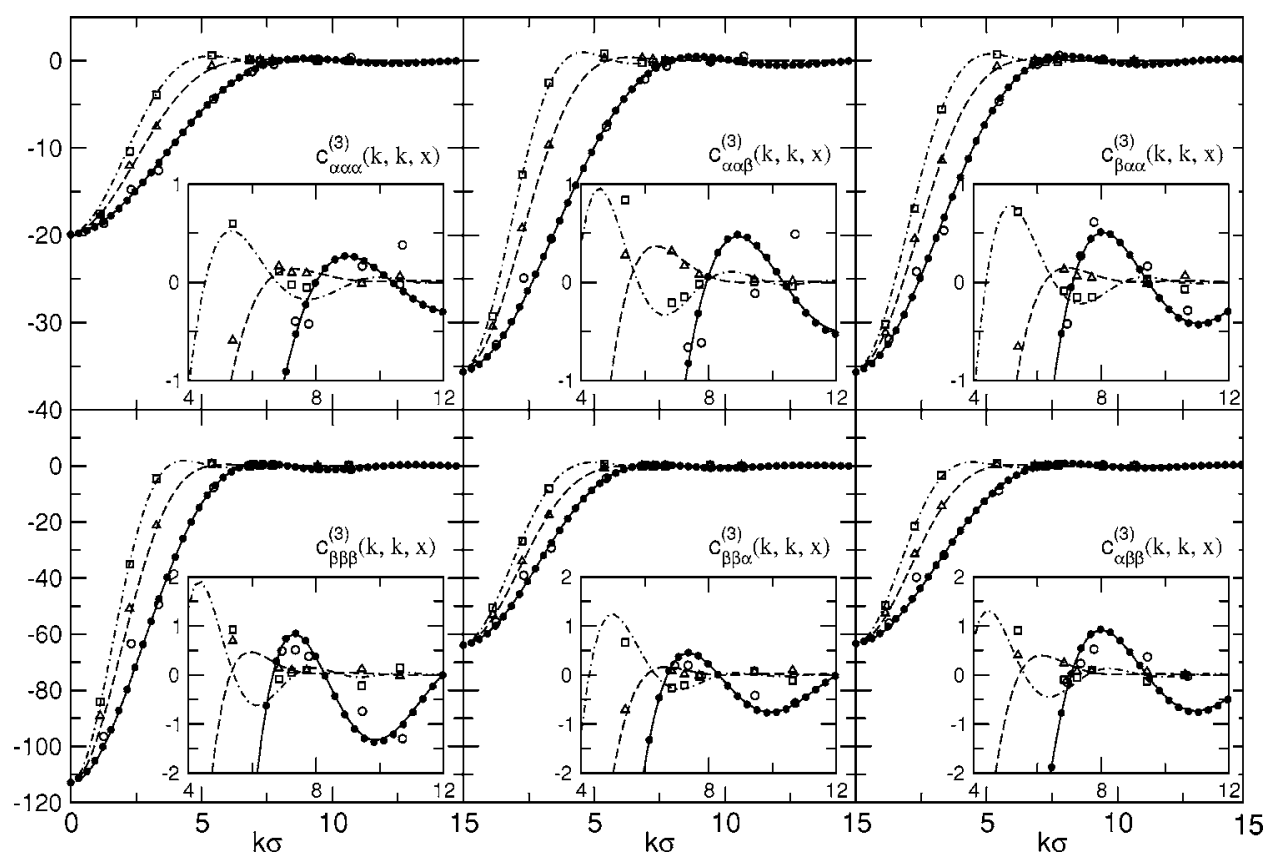

FIG. 10. $c_{\mu \nu \xi}^{(3)}(k, k, x)$ vs $k \sigma$ for an isosceles triangle configuration in a hard-sphere binary mixture and various configurations. Symbols correspond to simulation (open squares, $x$ $=1$ ), (up triangles, $x=0$ ), (open circles, $x=-1)$ and the sum rule Eq. (15) result for $x=-1$ (filled circles). Curves denote the BHP results. 
of the pair structure is removed, although at a considerable computational cost. On the other hand, the BHP ansatz can be used to provide approximations for the bridge function ${ }^{6}$ and thus yield an improved pair structure through an $\mathrm{OZ}$ formalism. For practical purposes, and taking into account the differences in the implementation of the two approaches one might conclude that the ISCVM is more suitable to provide information on the triplet distribution function in $r$-space, whereas Fourier transformed quantities and the triplet direct correlation itself are more amenable to be dealt with in terms of the BHP formalism.

\section{ACKNOWLEDGMENTS}

This work was funded by the Spanish Ministerio de Ciencia y Tecnología under Grant No. PB97-0258. S.J. acknowledges financial support from the Universidad Complutense de Madrid.

\section{APPENDIX: TREATMENT OF DISCONTINUITIES IN LEGENDRE TRANSFORMATIONS}

A discontinuous function $f_{\mu \nu \gamma}$ can be constructed in terms of $a_{\mu \nu}\left(r_{1}, r_{2}, \tau_{\mu \nu}\right)=\bar{h}_{\mu \nu \underline{\gamma}}^{(2)}\left(r_{1}, r_{2}, \tau_{\mu \nu}\right) \quad$ and $b_{\mu \nu}\left(r_{1}, r_{2}, \tau_{\mu \nu}\right)=\left(\partial h_{\mu \nu \underline{\gamma}}^{(2)}\left(r_{1}, r_{2}, x\right) / \partial x\right)_{x=\tau_{\mu \nu}}$, which represent, respectively, the value of the total correlation function and its first derivative at the contact angle $\left(\tau_{\mu \nu}=\cos \theta_{\mu \nu}\right)$, such that

$$
\begin{aligned}
f_{\mu \nu \underline{\gamma}}(x) & =0 \quad-1 \leqslant x<\tau_{\mu \nu} \\
& =a_{\mu \nu \underline{\gamma}}+b_{\mu \nu \underline{\gamma}}\left(x-\tau_{\mu \nu}\right) \quad \tau_{\mu \nu} \leqslant x \leqslant 1
\end{aligned}
$$

when added to $h_{\mu \nu \underline{\gamma}}^{(2)}\left(r_{1}, r_{2}, x\right)$ cancels its discontinuities at contact. Besides, Eq. (A1) has an analytic Legendre transform of the form,

$$
\begin{aligned}
\hat{f}_{n}^{\mu \nu} \underline{\gamma}= & \left(a_{\mu \nu}-b_{\mu \nu} \tau_{\mu \nu}\right) \frac{2 n+1}{2 n}\left[\tau_{\mu \nu} P_{n}\left(\tau_{\mu \nu}\right)-P_{n+1}\left(\tau_{\mu \nu}\right)\right] \\
& +\frac{b_{\mu \nu}}{2}\left\{\left[\frac{2 n+1}{n-1} \tau_{\mu \nu}^{2}-\frac{2 n+1}{(n-1)(n+2)}\right]\right. \\
& \left.\times P_{n}\left(\tau_{\mu \nu}\right)-\tau_{\mu \nu} \frac{(2 n+1)(n+1)}{(n-1)(n+2)} P_{n+1}\left(\tau_{\mu \nu}\right)\right\}
\end{aligned}
$$

for $n \geqslant 2$, and

$$
\begin{aligned}
& \hat{f}_{0}^{\mu \nu} \underline{\gamma}=\left(a_{\mu \nu}-b_{\mu \nu} \tau_{\mu \nu}\right)\left(1-\tau_{\mu \nu}\right) / 2+b_{\mu \nu}\left(1-\tau_{\mu \nu}^{2}\right) / 4, \\
& \hat{f}_{1}^{\mu \nu}=3\left(a_{\mu \nu}-b_{\mu \nu} \tau_{\mu \nu}\right)\left(1-\tau_{\mu \nu}^{2}\right) / 4+b_{\mu \nu}\left(1-\tau_{\mu \nu}^{3}\right) / 2
\end{aligned}
$$

for $n=0$ and 1 .

In a first step one then obtains a continuous and smooth function $h_{\mu \nu \underline{\gamma}}^{\prime(2)}$ by adding $f_{\mu \nu \underline{\gamma}}\left(h_{\mu \nu \underline{\gamma}}^{\prime(2)}=h_{\mu \nu \underline{\gamma}}^{(2)}+f_{\mu \nu \underline{\gamma}}\right)$ and then transforms numerically $h_{\mu \nu \gamma}^{\prime(2)}$ to get $\hat{h}_{n}^{\prime \mu \nu} \underline{\underline{\gamma}}$. Finally, the analytical transform of $f_{\mu \nu \underline{\gamma}}$ is subtracted to obtain the desired transform of the total correlation function $\left(\hat{h}_{n}^{\mu \nu} \underline{\underline{\gamma}}=\hat{h}_{n}^{\prime \mu \nu} \underline{\underline{\gamma}}\right.$ $\left.-\hat{f}_{n}^{\mu \nu} \underline{\underline{\gamma}}\right)$.

${ }^{1}$ J. Y. Raty, V. Godlevsky, Ph. Ghosez, C. Bichara, J. P. Gaspard, and J. R. Chelikowsky, Phys. Rev. Lett. 85, 1950 (2000).

${ }^{2}$ M. Lombardero, C. Martín, S. Jorge, F. Lado, and E. Lomba, J. Chem. Phys. 110, 1148 (1999).

${ }^{3}$ F. Sciortino and W. Kob, Phys. Rev. Lett. 86, 648 (2001).

${ }^{4}$ Y. Rosenfeld, D. Levesque, and J. J. Weis, J. Chem. Phys. 92, 6818 (1990).

${ }^{5}$ S. Jorge, G. Kahl, E. Lomba, and J. L. F. Abascal, J. Chem. Phys. 113, $3302(2000)$.

${ }^{6}$ J.-L. Barrat, J.-P. Hansen, and G. Pastore, Mol. Phys. 63, 747 (1988).

${ }^{7}$ S. Jorge, E. Lomba, and J. L. F. Abascal, J. Chem. Phys. 114, 3562 (2001).

${ }^{8}$ P. Attard, J. Chem. Phys. 91, 3072 (1989).

${ }^{9}$ E. Lomba, M. Álvarez, L. L. Lee, and N. G. Almarza, J. Chem. Phys. 104, 4180 (1996)

${ }^{10}$ M. Fushiki, Mol. Phys. 74, 307 (1991)

${ }^{11}$ J.-P. Hansen and I. R. McDonald, Theory of Simple Liquids, 2nd ed. (Academic, New York, 1986).

${ }^{12}$ D. G. Triezenberg and R. Zwanzig, Phys. Rev. Lett. 28, 1183 (1972).

${ }^{13}$ R. A. Lovett, C. Y. Mou, and F. P. Buff, J. Chem. Phys. 65, 570 (1976).

${ }^{14}$ R. Kjellander and S. Sarman, Mol. Phys. 70, 215 (1990).

${ }^{15}$ Y. Saad and M. H. Schultz, Technical Report No. 254, Yale University, 1993.

${ }^{16}$ P. H. Fries and M. Cosnard, J. Phys. (France) 48, 723 (1987).

${ }^{17}$ B. Bildstein and G. Kahl, J. Chem. Phys. 100, 5882 (1994). 